\title{
Application of Ion Sensitive Probe to High Density Plasmas in Magnum-PSI
}

\author{
Yuki HAYASHI, Noriyasu OHNO ${ }^{1)}$, Hennie van der MEIDEN ${ }^{2)}$, John SCHOLTEN ${ }^{2)}$, \\ Shin KAJITA ${ }^{3)}$, Jonathan van den BERG $^{2)}$, Renato PERILLO ${ }^{2)}$, \\ Jordy VERNIMMEN ${ }^{2)}$ and Thomas MORGAN ${ }^{2)}$ \\ National Institute for Fusion Science, National Institutes of National Sciences, Toki 509-5292, Japan \\ ${ }^{1)}$ Graduate School of Engineering, Nagoya University, Nagoya 464-8603, Japan \\ ${ }^{2)}$ DIFFER-Dutch Institute for Fundamental Energy Research, Eindhoven, The Netherlands \\ ${ }^{3)}$ Institute of Materials and Systems for Sustainability, Nagoya University, Nagoya 464-8603, Japan
}

(Received 2 May 2019 / Accepted 21 June 2019)

\begin{abstract}
Feasibility of an ion sensitive probe was evaluated in high electron density $\left(>5 \times 10^{19} \mathrm{~m}^{-3}\right)$ helium plasmas produced in the Magnum-PSI device. The ion sensitive probe showed that the ion temperature was $\sim 1 \mathrm{eV}$ and almost equal to the electron temperature. Increasing the neutral pressure to efficiently lead to the electron-ion recombination processes, the electron currents were collected at the ion collector. A secondary electron emission in the guard electrode might have an effect on the ion current.
\end{abstract}

(c) 2019 The Japan Society of Plasma Science and Nuclear Fusion Research

Keywords: ion sensitive probe, ion temperature, $I-V$ characteristic, Magnum-PSI

DOI: $10.1585 /$ prr.14.1202135

Plasma detachment is realized by means of volumetric recombination processes in front of the divertor targets in magnetically confined fusion devices. Electron-ion recombination (EIR) processes dominate when the electron temperature, $T_{\mathrm{e}}$, is less than $1 \mathrm{eV}$, and the rate coefficients of molecular activated recombination (MAR) processes have a peak at $T_{\mathrm{e}} \sim 1-2 \mathrm{eV}$ [1]. Contribution of ion temperature, $T_{\mathrm{i}}$, is considerable on the cooling process of electrons on such a low temperature plasma. This is because the electron-neutral interactions such as electron impact ionization and excitation are less dominant than $T_{\mathrm{e}}-T_{\mathrm{i}}$ relaxation process when $T_{\mathrm{e}}<5 \mathrm{eV}$. The insufficient collision between ions and neutral particles discourages a reduction in $T_{\mathrm{i}}$. As a result, $T_{\mathrm{i}}$ determines a lower limit of $T_{\mathrm{e}}$.

Energy balance among electron, ion and neutral particles can be investigated in detail by utilizing the steady state plasma generated in linear plasma devices. The ion temperature was measured with an ion sensitive probe (ISP) [2] in some linear plasma devices [3,4]. In this Rapid Communication, the purpose is to investigate feasibility of an ISP application to high electron density, $n_{\mathrm{e}}$, and high neutral pressure, $P_{\mathrm{n}}$, plasma in Magnum-PSI [5], where the parameters are relevant to divertor region in ITER.

Magnum-PSI generated a steady-state He plasma. In order to measure $T_{\mathrm{i}}$ in the case that EIR processes are dominant, plasma-neutral interactions were enhanced by means of additional He puffing in target chamber, hence increasing the He neutral pressure, $P_{\mathrm{n}}$. The magnetic field of $0.3 \mathrm{~T}$, which was produced by superconducting coils, provided confinement of the plasma. Because the ion cyclotron frequency was greater than the ion-neutral collision frequency, we could assume the magnetized plasma. The plasma column was terminated with a target plate. It is expected that $T_{\mathrm{i}}$ and $T_{\mathrm{e}}$ are in the range of $0.5-2 \mathrm{eV}$ in front of the target plate. Under these conditions, the ion and electron Larmor radii are $0.8-1.5 \mathrm{~mm}$ and $9-18 \mu \mathrm{m}$, respectively. The Larmor radius is an important parameter in the application of the ISP to magnetized plasma. Figure 1 (a) shows the schematic of the ISP. The ISP consists of a rod-shaped ion collector and a cylindrical guard electrode surrounding the ion collector. The top surface of the ion collector is located deeply from that of the guard electrode to avoid inflow of electrons and to collect only ions at the ion collector. In the present study, the distance between the top surfaces of two electrodes (denoted by $h$ in Fig. 1 (a)) was set to be $0.5 \mathrm{~mm}$. The ISP was vertically inserted into the plasma column and measurements were performed at a radial position, $r$, of $7 \mathrm{~mm}$ and with the distance of $80 \mathrm{~mm}$ from the target. The diameters of the ion collector and guard electrode were, respectively, 0.5 and $1.5 \mathrm{~mm}$, which were selected not to disturb the plasma column with the diameter of $20 \mathrm{~mm}$.

Figures 1 (b)-(d) show the current-voltage ( $I-V)$ characteristics of the ion collector and the guard electrode in the ISP. Here, $I_{\mathrm{p}}$ and $I_{\mathrm{g}}$ denote, respectively, the currents into the ion collector and the guard electrode, which are at the voltages of $V_{\mathrm{p}}$ and $V_{\mathrm{g}}$, respectively; $V_{\mathrm{p}}$ was negatively higher than $V_{\mathrm{g}}$ by $1.5 \mathrm{~V}$ to avoid an electron current into the ion collector. Figure 1 (b) shows the $I$ - $V$ characteristics 
(a)
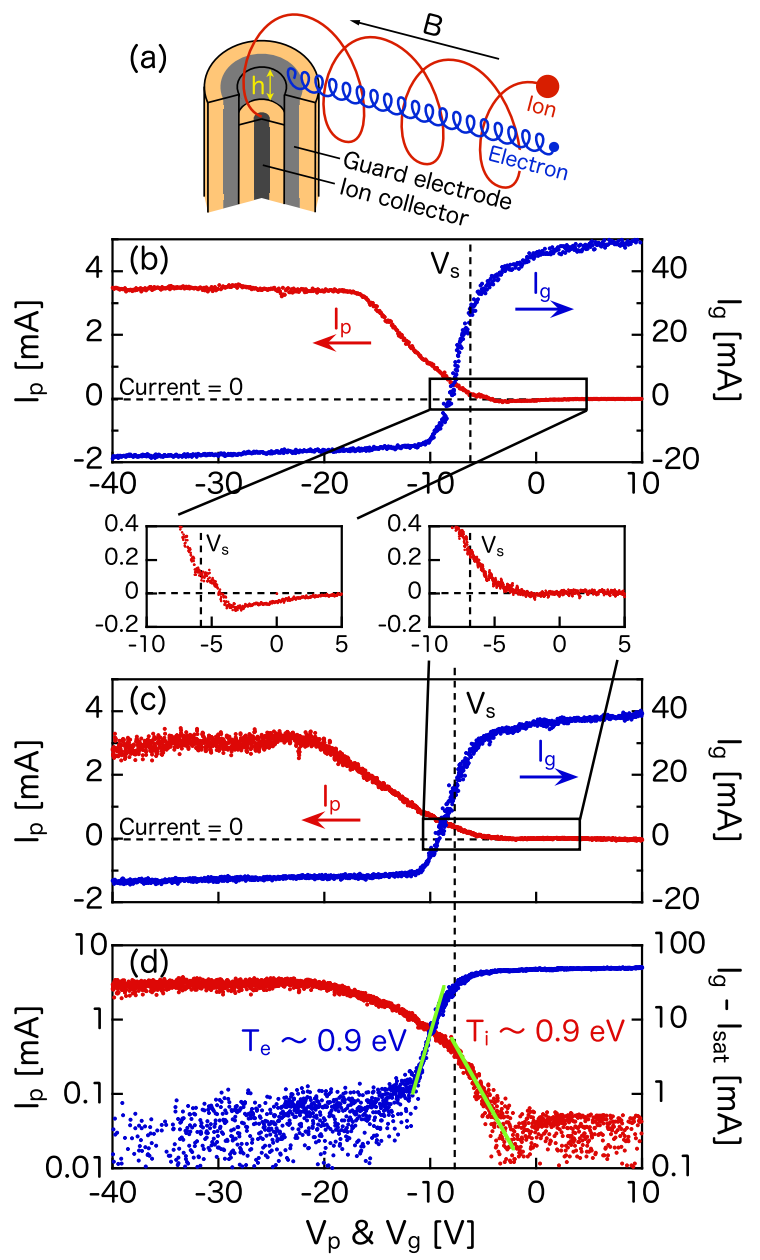

Fig. 1 (a) Schematic of ISP. (b) $I-V$ characteristics of the ISP $\left(I_{\mathrm{d}}=160 \mathrm{~A}\right.$, and $\left.P_{\mathrm{n}} \sim 1.3 \mathrm{~Pa}\right)$. (c) Linear and (d) logarithmic plots of $I_{\mathrm{p}}$ and $I_{\mathrm{g}}\left(I_{\mathrm{d}}=140\right.$ A and $\left.P_{\mathrm{n}} \sim 0.3 \mathrm{~Pa}\right)$.

when the discharge current, $I_{\mathrm{d}}$, of $160 \mathrm{~A}$ and $P_{\mathrm{n}} \sim 1.3 \mathrm{~Pa}$. When $V_{\mathrm{p}} \sim$ the space potential, $V_{\mathrm{s}}$, electron currents were collected in the $I_{\mathrm{p}}$ curve, meaning that the separate collection of ions in the ion collector failed. In the experiments performed in PSI-2 [3], similar unanticipated negative currents were found when $V_{\mathrm{p}} \geqq V_{\mathrm{g}}$. The problem was caused by the electrons moving by $\boldsymbol{E} \times \boldsymbol{B}$ drift along the equipotential surface that intersects with the ion collector [6]. However, this was not the case in the present study, because $V_{\mathrm{p}}<V_{\mathrm{g}}$ was ensured.

A reason for making $I_{\mathrm{p}}$ negative could be the influence of secondary electron emission in the guard electrode. The Refs. $[7,8]$ explained that the second electron current from the probe surface was induced by metastable He flux. Some of the secondary emitted electrons from the guard electrode can reach the ion collector, leading to the negative current observed in $I_{\mathrm{p}}$. The second electron current was small and not seen in $I_{\mathrm{g}}$ curve, whereas the impact was not negligible regarding $I_{\mathrm{p}}$ because of the small ion current. Although the present pressure was much lower than the cases in $[7,8]$, there should be many metastable

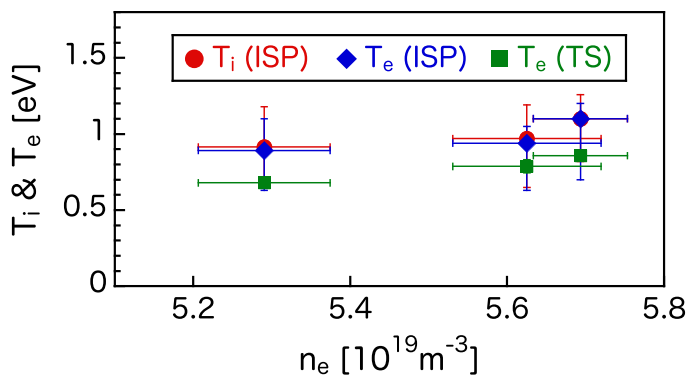

Fig. 2 Scatter plots of $T_{\mathrm{e}}$ and $T_{\mathrm{i}}$ vs. $n_{\mathrm{e}}$ under three different discharge conditions. $\left(P_{\mathrm{n}} \sim 0.3 \mathrm{~Pa}\right)$.

atoms in the present experiments, as well, because the metastable states can be produced by EIR processes at the present $T_{\mathrm{e}}$ [9]. The effect of metastable atoms on the ISP measurement should be investigated by comparative experiments with different source gas which does not include metastable.

Figures 1 (c)-(d) show typical $I-V$ characteristics of the ISP when $T_{\mathrm{i}}$ can be analyzed. The $I-V$ characteristics were obtained at $I_{\mathrm{d}}$ of $140 \mathrm{~A}$ and $P_{\mathrm{n}}$ of $0.3 \mathrm{~Pa}$. The inflow of electrons into the ion collector was not observed when $V_{\mathrm{p}}$ was greater than $V_{\mathrm{s}}$ in the $I_{\mathrm{p}}$ curve, meaning that only ions were successfully collected separately from electrons. The logarithmic plots of $I_{\mathrm{p}}$ and $I_{\mathrm{g}}$ after the subtraction of the ion saturation current, $I_{\text {sat }}$, give us $T_{\mathrm{i}} \sim T_{\mathrm{e}} \sim 0.9 \mathrm{eV}$ at the same time. Here, the fitting ranges for $T_{\mathrm{i}}$ and $T_{\mathrm{e}}$ were selected when $V_{\mathrm{p}}$ and $V_{\mathrm{g}}$ were, respectively, higher and lower than $V_{\mathrm{s}}$ and when $I_{\mathrm{p}}$ and $I_{\mathrm{g}}$ showed exponential decay.

Figure 2 shows $n_{\mathrm{e}}$ dependences of $T_{\mathrm{i}}$ and $T_{\mathrm{e}}$. In order to observe $n_{\mathrm{e}}$ dependence, $I_{\mathrm{d}}$ was varied from 140 to $160 \mathrm{~A}$ at the $P_{\mathrm{n}}$ of $0.3 \mathrm{~Pa}$. Thomson scattering (TS) measurement at the ISP position was performed to obtain $n_{\mathrm{e}}$ and $T_{\mathrm{e}}$. The measured $T_{\mathrm{e}}$ by TS measurement is also shown in Fig. 2. A systematic discrepancy between ISP and TS were found. The validation of ISP diagnostics in recombining plasma is necessary in detail by a quantitative comparison with the collective TS [10] and the TS in the future. Slight increases in $T_{\mathrm{e}}$ and $T_{\mathrm{i}}$ with $n_{\mathrm{e}}$ were due to the increase in discharge power. Thermalization between ions and electrons can easily occur, because the density is high in the Magnum-PSI device. Roughly, the estimated $T_{\mathrm{i}}-T_{\mathrm{e}}$ relaxation time was of the order of $\mu \mathrm{s}$, whereas typical ion resident time estimated with the plasma length and ion sound velocity was of the order of $100 \mu \mathrm{s}$. Therefore, thermal equilibrium between ions and electrons can be assumed at the target region.

This work was supported by Japan Society for the Promotion of Science KAKENHI (16H02440), Grant-in-Aid for JSPS Research Fellow (17J05222).

[1] A.Yu. Pigarov et al., Phys. Lett. A 222, 251 (1996).

[2] I. Katsumata et al., Jpn. J. Appl. Phys. 6, 123 (1967). 
[3] N. Ezumi et al., J. Nucl. Mater. 337-339, 1106 (2005).

[4] H. Takahashi et al., Phys. Plasmas 26, 022511 (2019).

[5] J. Rapp et al., Fusion Eng. Des. 85, 1455 (2010).

[6] N. Ezumi, Contrib. Plasma Phys. 41, 488 (2001).
[7] D. Korzec et al., Sci. Technol. Adv. Mater. 2, 595 (2001).

[8] M.R. Talukder et al., J. Appl. Phys. 91, 9529 (2002).

[9] S. Kajita et al., Phys. Plasmas 24, 073301 (2017).

[10] H.J. Meiden et al., Appl. Phys. Lett. 109, 261102 (2016). 